
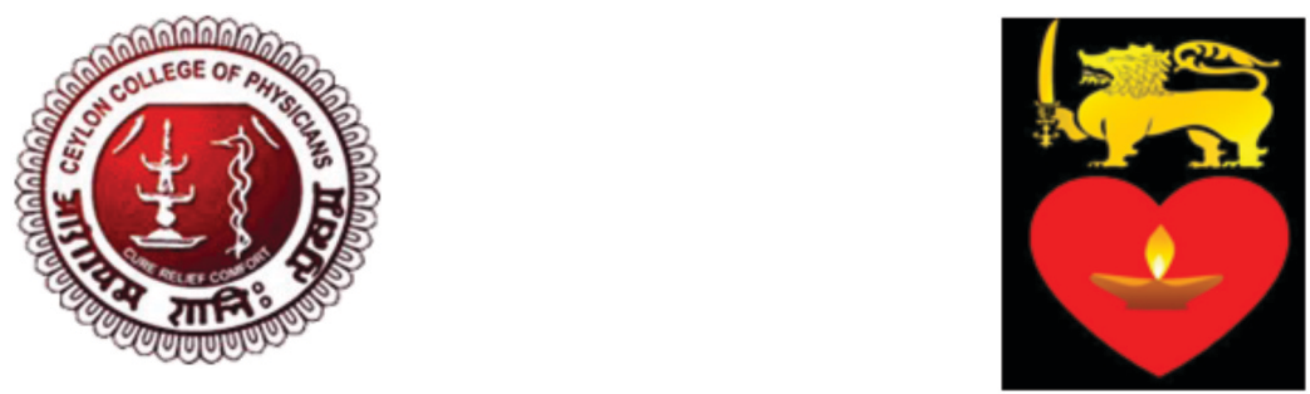

\title{
STEMI Management Guidelines
}

Compiled by Sri Lanka Heart Association $\&$ Sri Lanka STEMI Forum

Journal of the Ceylon College of Physicians Vol 452014 Supplement 


\section{STEMI Management Guidelines}

\section{CONTENTS}

Page No.

1.0 Overall System Goals S2

2.0 Diagnosis

3.0 Acute Phase Management

4.0 Reperfusion at PCl-capable (Tertiary Care) Hospitals

5.0 Reperfusion at Non-PCl capable Hospitals (All levels)

S7

6.0 Periprocedural Antiplatelet and Antithrombotic Treatment

7.0 Assessment of Reperfusion and discharge

10.0 Other Standard General and/or Long-Term Therapeutic Measures of ST Segment Elevation Myocardial Infarction 


\subsection{Overall System Goals}

The goal of STEMI care is:

- To keep total ischemic time within 120 minutes (ideally 60 minutes).

- For thrombolysis Door to Needle (D2N) time to be 30 minutes.

- For primary PCI Door to Balloon (D2B) time to be 90 minutes.

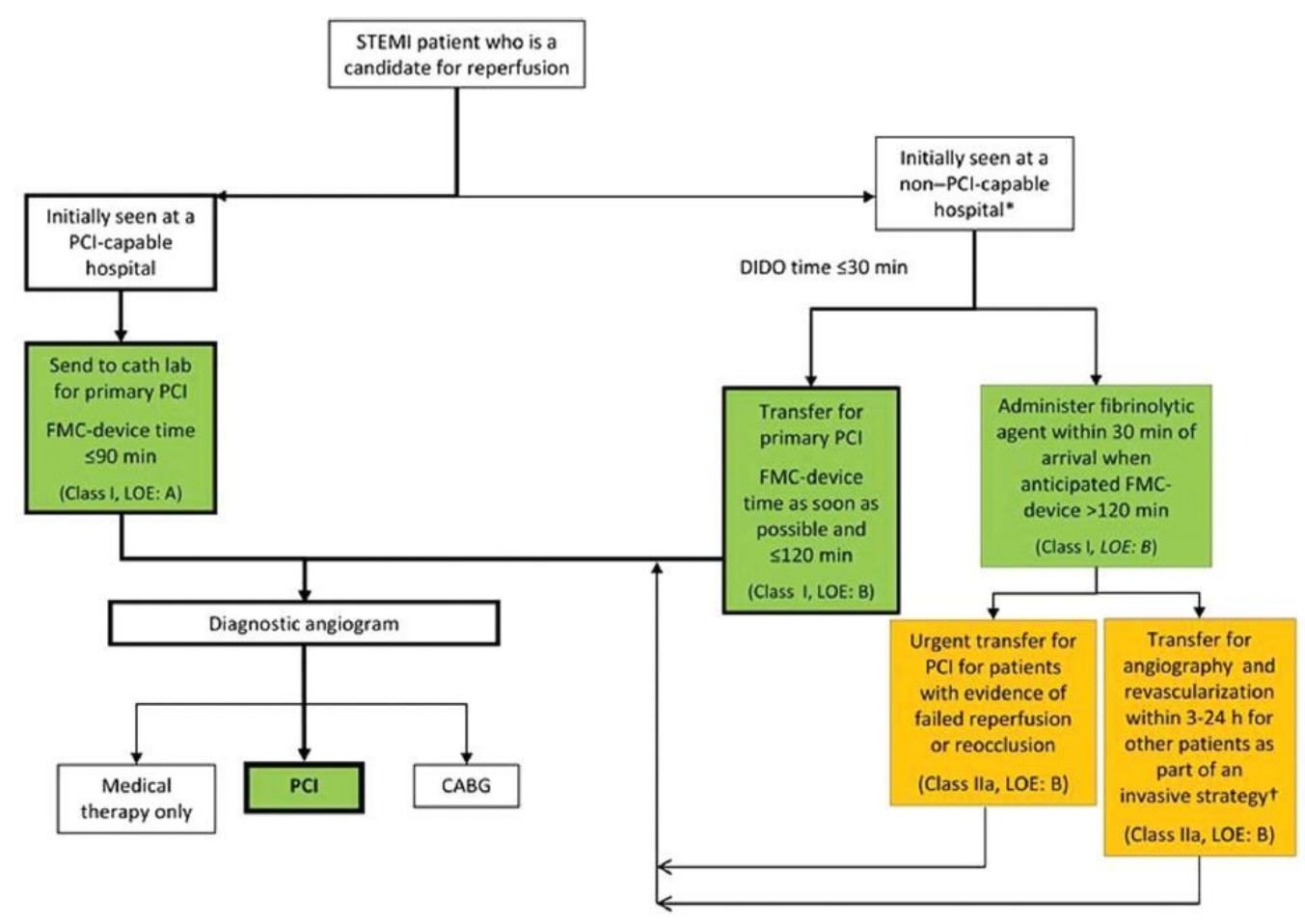

Reperfusion Therapy for Patients with STEMI

\subsection{Diagnosis}

\section{Initial Diagnosis of Acute Myocardial Infarction (AMI)}

- ECG Monitoring should be initiated as soon as possible in all patients with suspected STEMI

- A 12 lead ECG should be obtained and interpreted as soon as possible ideally at the point of First Medical Contact (FMC) (target delay $\leq 10$ minutes)

- Blood sampling for serum markers should be routinely done in the acute phase but initiation of treatment should not be delayed until results are available

- Echocardiography may assist in making the diagnosis in uncertain cases but should not delay treatment initiation

- In the presence of LBBB ECG diagnosis of AMI is difficult, but often possible if marked ST abnormalities are present. 


\section{ECG Diagnosis of ST segment Myocardial Infarction (STEMI)}

- ST segment elevation is measured at $\mathrm{J}$ point

- In men below 40 years of age it should be $\geq 0.25 \mathrm{mV}$

- It should be present in 2 contiguous leads

- $0.2 \mathrm{mV}$ in men over the age of 40 years

- $\quad \geq 0.15 \mathrm{mV}$ in women in leads $\mathrm{V} 2-\mathrm{V} 3$ and/or $\geq 0.1 \mathrm{mV}$ in other leads

- Additional recordings of, for example lead V7, V8 and V9 ( $\geq 0.05 \mathrm{mV})$ may be helpful to make the diagnosis in selected cases when infero-basal myocardial infarction is suspected due to circumflex occlusion.

\section{Atypical Presentations}

- LBBB

- Ventricular paced rhythm

- Persistent ischemic symptoms in the absence of diagnostic ST segment elevation

- Isolated Posterior MI

- ST segment elevation in lead aVR

- A high level of suspicion for myocardial infarction must be maintained in women, diabetics and elderly patients with atypical symptoms.

\section{Clinical Risk Assessment}

Indicators of high risk in the acute phase:

- Older age

- Fast heart rate

- Hypotension

- Killip class $>1$

- Anterior infarction

- Previous infarction

- Elevated initial serum creatinine

- History of heart failure

\subsection{Acute Phase Management}

\section{In-Hospital Acute Phase Management}

- Whenever possible STEMI patients should be admitted to a Coronary Care Unit (CCU)

- Ready access to facilities for hemodynamic monitoring and defibrillation should be available. 
- All teaching and tertiary care level hospitals with catheterization labs and facilities for reperfusion, participating in the care of STEMI patients, should ideally have a coronary care unit (CCU) equipped to provide all aspects of care for STEMI patients, including treatment of ischemia, severe heart failure, arrhythmia and common comorbidities.

\section{Oxygen}

- Continuous monitoring of ECG (for ST deviation and any arrhythmias) and Pulse Oxymetry

- Oxygen at $2 \mathrm{~L} /$ minute administered via nasal cannula for the first 6 hours

- Continued thereafter if oxygen saturation is below $90 \%$

\section{Access}

- Intravenous (IV) access is mandatory

- Start a running infusion to keep the veins open

- A second IV line is needed if medication is to be given

\section{Pain Management}

- Patients with ongoing ischemic discomfort should receive sublingual nitroglycerin $(0.4 \mathrm{mg})$ every 5 minutes for a total of 3 doses

- After that intravenous nitroglycerin should be considered

- Morphine sulphate (2 to $4 \mathrm{mg} \mathrm{IV}$ ) is the analgesic of choice in STEMI care

- It could be repeated at 5 to 15 minutes intervals with increment of 2 to $8 \mathrm{mg}$ IV

- Patients who are routinely taking NSAIDs (except Aspirin) should have them stopped to reduce mortality and prevent complications

\section{Blood for investigations}

- Blood to be drawn for testing:

o Serum Cardiac Biomarkers

o FBC including platelet

o Prothrombin Time with INR

o Activated Partial Thromboplastin Time (aPTT)

o Electrolytes

○ Magnesium

o Blood Urea Nitrogen (BUN)

o Creatinine 
o Glucose

o Lipid profile: (see under lipid management below)

- Total cholesterol

- Low density lipoprotein (LDL) cholesterol - since LDL levels tend to decrease the first days after myocardial infarction they are best measured as soon as possible after admission

- High density lipoprotein (HDL) cholesterol

- Fasting triglycerides

\section{Imaging}

- Emergency Echocardiography:

o In the acute phase when the diagnosis is in doubt emergency echocardiography may be useful

- Emergency angiography:

o If emergency echocardiography is unavailable or inconclusive while the diagnosis is still in doubt emergency angiography should be considered

Reperfusion (see under specific reperfusion strategies below for details)

- Should not wait for lab results to implement reperfusion strategy

- All patients with symptoms of $<12$ hours duration and ST segment elevation or (presumably) LBBB (Left Bundle Branch Block)

- If there is evidence of ischemia, even if symptoms may have started $>12$ hours ago (preferably primary $\mathrm{PCl}$ )

- In those who have stuttering symptoms and ECG changes (preferably primary PCI)

\section{Monitoring}

- Monitoring of vital signs hourly till the patient is stable/every 4 hourly when stabilized

- No oral feeds except for sips of water until stable

- Strict bed rest during acute phase

\section{Stabilized patient}

- Patients undergoing uncomplicated successful reperfusion therapy:

o should be kept in the CCU for a minimum of 24 hours

o After that may be moved to a step down monitored bed for another 24 to 48 hours

- Or when the patient is stable clinically for $12-24$ hours she may be transferred to a ward/step down unit.

- Bedside chair and a commode could be allowed for the stable patients' to use 


\subsection{Reperfusion at a PCl-capable hospital}

Teaching and tertiary level hospitals with cath labs:

1. Cardiology Institute of NHSL, Colombo

2. Sri Jayewardenepura Teaching Hospital, Kotte

3. Teaching Hospital Kandy

4. Teaching Hospital Kurunegala

5. Teaching Hospital Jaffna

6. Teaching Hospital Karapitiya

- Primary $\mathrm{PCl}$ is the preferred reperfusion strategy

- It is defined as percutaneous catheter intervention in the setting of STEMI, without previous thrombolytic therapy.

\section{Primary PCl}

\section{System goals:}

- Should be performed expeditiously by a well-experienced team

- Ideally should be initiated promptly within 90 minutes of door to balloon time

- If presented early with a large area or myocardium at risk and/or at primary PClenabled hospitals, the goal should be to achieve a door to balloon time of $\leq 60$ minutes

- However, it is acceptable for primary PCI if the anticipated delay is $\leq 120$ (door to balloon time) minutes, when the first presentation is to a non- $\mathrm{PCl}$ capable hospital and even if thrombolysis therapy could be initiated within the recommended 30 minutes of door to needle time.

\section{Primary PCI should be performed:}

- In all patients with STEMI and ischemic symptoms of less than 12 hours' duration.

- In patients with STEMI and ischemic symptoms of less than 12 hours' duration who have contraindications to fibrinolytic therapy, irrespective of the time delay from FMC.

- In patients with STEMI and cardiogenic shock or acute severe HF, irrespective of time delay from symptom onset.

\section{Primary $\mathrm{PCl}$ is reasonable to be performed:}

- In patients with STEMI if there is clinical and/or ECG evidence of ongoing ischemia between 12 and 24 hours after symptom onset

\section{PCI should not be performed:}

- In a non-infarct artery at the time of primary $\mathrm{PCI}$ in patients with STEMI who are hemodynamically stable

- In a totally occluded artery > 24 hours after symptom onset in stable patients with no signs of ischemia 


\section{Placement of Stents:}

- Placement of a stent is useful and recommended in primary $\mathrm{PCl}$ for patients with STEMI, as opposed to angioplasty without stenting.

- Stenting could be either Bare Metal Stent (BMS) or Drug Eluting Stent (DES)

- BMS should be used in patients with high bleeding risk, inability to comply with 1 year of Dual Anti-Platelet Therapy (DAPT), or anticipated invasive or surgical procedures in the next year

- DES should not be used in primary PCI for patients with STEMI who are unable to tolerate or comply with a prolonged course of DAPT because of the increased risk of stent thrombosis with premature discontinuation of one or both agents.

- If the patient has no contraindications to prolonged DAPT and is likely to be compliant, DES should be preferred over BMS.

- Balloon angioplasty without stent placement may be used in selected patients.

\section{Other procedural aspects of Primary PCI}

- Aspiration thrombectomy: manual aspiration thrombectomy is reasonable for patients undergoing primary $\mathrm{PCl}$

- If performed by an experienced radial operator, radial access should be performed over femoral access

- Routine use of distal protection device is not recommended

- Routine use of IABP (in patients without shock) is not recommended

\subsection{Reperfusion at a Non-PCI capable Hospital}

Teaching and tertiary hospitals with no cath labs, provincial and district general hospitals, base hospitals and any other centres where STEMI is managed

- If no contraindications thrombolytic therapy should be given when there is an anticipated delay to performing primary $\mathrm{PCl}$ more than 120 minutes of FMC

- The goal is to achieve a door to needle time of less than 30 minutes

\section{Thrombolysis}

\section{Doses of thrombolytic Agents:}

Streptokinase (SK)

- 1.5 million units over 30 to 60 minutes IV

- Specific contraindications: Prior SK or anistreplase

Tenecteplase (TNK)

- Single IV bolus

- $30 \mathrm{mg}$ if $<60 \mathrm{~kg}$ 
- $\quad 35 \mathrm{mg}$ if $60-70 \mathrm{~kg}$

- $40 \mathrm{mg}$ if $70-80 \mathrm{~kg}$

- $45 \mathrm{mg}$ if $80-90 \mathrm{~kg}$

- $50 \mathrm{mg}$ if $\geq 90 \mathrm{~kg}$

\section{Contraindications for thrombolysis:}

\section{Absolute contraindications:}

- Previous Intra-Cranial Hemorrhage (ICH)

- Stroke of unknown origin at any time

- Ischemic stroke in the past 6 months

- Central Nervous System damage

- Malignant intracranial neoplasm

- Other intracranial lesions (aneurysm or AV malformation)

- History of closed head or facial trauma within 3 months

- Major trauma/surgery within preceding 3 weeks

- Suspected aortic dissection

- Active bleeding or known bleeding diathesis (excluding menses)

- Gastrointestinal bleeding within the past month

- Non-compressible punctures in the past 24 hours (Eg: liver biopsy, lumber puncture)

\section{Relative contraindications:}

- Previous ischemic stroke beyond 12 months

- Transient ischemic attack in the preceding 6 months

- Oral anticoagulation therapy

- Pregnancy or within 1 week postpartum

- Recent (2-4 weeks) internal bleeding

- Prolonged or traumatic CPR

- Active peptic ulcer (only if the ulcer is actively bleeding; if only stool occult blood positivity, may be considered for thrombolysis)

- Severe uncontrolled hypertension (SBP >180mm Hg and/or DBP $110 \mathrm{~mm} \mathrm{Hg}$ )

- Patients presenting with hypertension should be administered beta blockers, nitroglycerin and analgesics promptly to lower blood pressure and reduce risk of $\mathrm{ICH}$ following thrombolysis

- Patients on warfarin therapy have higher rates of hemorrhage - higher the INR, higher the risk

- Advanced liver disease

- Infective endocarditis 
- Hemorrhagic diabetic retinopathy is not a contraindication for thrombolysis

- Change in the neurological status especially 24 hours following reperfusion should be considered to be due to $\mathrm{ICH}$ until proven otherwise.

\section{Indications for thrombolytic therapy:}

- In the absence of contraindications, thrombolytic therapy should be given to patients with STEMI and onset of ischemic symptoms within the previous 12 hours when it is anticipated that primary $\mathrm{PCl}$ cannot be performed within 120 minutes of FMC.

- In the absence of contraindications and when $\mathrm{PCl}$ is not available, thrombolytic therapy is reasonable for patients with STEMI if there is clinical and/or ECG evidence of ongoing ischemia within 12 to 24 hours of symptom onset and a large area of myocardium at risk or hemodynamic instability.

- A fibrin specific agent (tenecteplase, alteplase and reteplase) is recommended over non-fibrin specific agents (streptokinase)

- Oral or IV aspirin must be administered

- Clopidogrel is indicated in addition to aspirin

- Transfer to PCl capable hospital is indicated all patients after thrombolysis

- Thrombolytic therapy should not be administered to patients with ST depression except when a true posterior (inferobasal) MI is suspected or when associated with ST elevation in lead aVR.

\subsection{Periprocedural Antiplatelet and Antithormbotic Treatment}

\section{When no reperfusion therapy Available \\ (Medical Therapy at Primary Care Level)}

Doses of antiplatelet and antithrombin co-therapies:

\section{Antiplateletes:}

- Aspirin - starting dose 150-500 mg orally

- 75 mg/day orally

\section{Antithrombin:}

- Unfractionated heparin:

o $60 \mathrm{U} / \mathrm{kg}$ IV bolus with maximum of $4000 \mathrm{U}$ followed by an IV infusion of $12 \mathrm{U} / \mathrm{Kg}$ with a maximum of $1000 \mathrm{U} /$ hours for 24 to 48 hours

o Target aPTT: 50 to 70 s or 1.5 to 2.0 times that of control to be monitored at $3,6,12$ and 24 hours

\section{- Enoxaparin:}

o In patients $<75$ years of age: 
- $30 \mathrm{mg}$ IV bolus followed 15 minutes later by $1 \mathrm{mg} / \mathrm{kg}$ SC every 12 hours until hospital discharge or maximum of 8 days.

- The first two doses should not exceed $100 \mathrm{mg}$

o In patients $>75$ years of age:

- No IV bolus.

- Start with first SC dose of $0.75 \mathrm{mg} \mathrm{kg}$ with a maximum of $75 \mathrm{mg}$ for the first two SC doses

o In patients with creatinine clearance of $<30 \mathrm{~mL} / \mathrm{min}$ regardless of age the SC doses are given once every 24 hours.

\section{- Fondaparinux:}

○ $2.5 \mathrm{mg}$ IV bolus

o Followed by a SC dose of $2.5 \mathrm{mg}$ once daily up to 8 days or hospital discharge

\section{In Primary PCI}

Those patients undergoing primary $\mathrm{PCl}$ should receive a combination of DAPT (Dual Antiplatelet Therapy) with aspirin and an adenosine diphosphate (ADP)-receptor blocker, as early as possible before angiography, and a parenteral anticoagulant.

\section{Antiplatelet therapy:}

- Aspirin oral

- if unable to swallow IV

- ADP-receptor blockers:

o Prasugrel in clopidogrel-naïve patients, if there's no history of prior stroke/TIA and the age is $<75$ years.

o Tricagrelor

- Clopidogrel preferably when prasugrel or tricagrelor are either not available or contraindicated

- GP IIb/lla inhibitors should be considered for bail-out therapy if there is angiographic evidence of massive thrombus, slow or no-reflow or a thrombotic complication

- Routine use of GP IIb/Illa inhibitor as an adjunct to primary PCI performed with unfractionated heparin may be considered in patients without contraindications.

- Upstream use of GP Ilb/Illa inhibitor (vs in lab use) may be considered in high risk patients undergoing transfer for primary $\mathrm{PCl}$

- GP Ilb/llla inhibitors:
o Abciximab
- Eptifibatide (with double bolus)
o Tirofiban (with a high bolus dose) 


\section{Anticoagulants:}

- An injectable anticoagulant must be used

- Bivalirudin is recommended over unfractionated heparin and a GP IIb/llla blocker

- Enoxaparin (with or without GP Ila/lllb blocker) may be preferred over unfractionated heparin

- Unfractionated heparin with or without routine GP lla/lllb blocker must be used in patients not receiving bivalirudin or enoxaparin

- Fondaparinux is not recommended for primary $\mathrm{PCI}$

- The use if fibrinolysis before planned primary $\mathrm{PCl}$ is not recommended

\section{Doses of antiplatelet and antithrombin co-therapies:}

\section{Antiplateletes:}

- Aspirin:

o loading dose of $150-500$ mg orally

o Or $80-150 \mathrm{mg} \mathrm{IV}$ if oral ingestion is not possible

o Followed by a maintenance dose $75-100 \mathrm{mg} /$ day

- Clopidogrel:

o Loading dose of $600 \mathrm{mg}$ orally

o Followed by a maintenance dose of $75 \mathrm{mg} /$ day

- Prasugrel:

o Loading dose of $60 \mathrm{mg}$ orally

o Followed by a maintenance dose of $10 \mathrm{mg} /$ day

o In patients with body weight $<60 \mathrm{~kg}$, a maintenance dose of $5 \mathrm{mg}$ is recommended

o In patients $>75$ years, prasugrel is generally not recommended, but a dose of $5 \mathrm{mg}$ should be used if treatment is deemed necessary

- Ticagrelor:

o Loading dose of $180 \mathrm{mg}$ orally

o Followed by a maintenance dose of $90 \mathrm{mg} \mathrm{BID}$

- Abciximab:

o Bolus of $0.25 \mathrm{mg} / \mathrm{kg} \mathrm{IV}$ and $0.125 \mu \mathrm{g} / \mathrm{kg} / \mathrm{min}$ infusion (maximum $10 \mu \mathrm{g} / \mathrm{min}$ ) for 12 hours

- Eptifabatide:

o Double bolus of $180 \mu \mathrm{g} / \mathrm{kg}$ IV (given at a 10 minutes interval) followed by an infusion of $2.0 \mu \mathrm{g} / \mathrm{kg} / \mathrm{min}$ for 18 hours 
- Tirofiban:

- $25 \mu \mathrm{g} / \mathrm{kg}$ over 3 minutes IV

o Followed by a maintenance infusion of $0.15 \mu \mathrm{g} / \mathrm{kg} / \mathrm{min}$ for 18 hours

\section{Antithrombin:}

- Unfractionated Heparin:

o 70-100 U/kg IV bolus when no GP Ilb/llla inhibitor is planned

o $50-60 \mathrm{U} / \mathrm{kg}$ IV bolus with GP Ilb/Illa inhibitors

- Enoxaparin:

○ $0.5 \mathrm{mg} / \mathrm{kg}$ IV bolus

- Bivalirudin:

o $0.75 \mathrm{mg} / \mathrm{kg}$ IV bolus followed by IV infusion of $1.75 \mathrm{mg} / \mathrm{kg} / \mathrm{h}$ for up to 4 hours after the procedure as clinically warranted

o After cessation of the $1.75 \mathrm{mg} / \mathrm{kg} / \mathrm{h}$ infusion, a reduced infusion dose of 0.25 $\mathrm{mg} / \mathrm{kg} / \mathrm{h}$ may be continued for $4-12$ hours as clinically necessary

\section{In Thrombolytic Therapy}

\section{Antithrombin Co-therapy with fibrinolysis:}

- Anticoagulation is recommended in STEMI patients treated with lytics until revascularization (if performed)

- Or else for 48 hours or for the duration of hospital stay up to 8 days

- The anticoagulant can be:

o Enoxaparin IV followed by SC preferred over UFH

o UFH is given as a weight adjusted IV bolus and infusion

- In patients treated with streptokinase, fondaparinux IV bolus followed by SC dose 24 hours later.

- UFH was found to improve coronary patency after alteplase but not after streptokinase

\section{Doses of antiplatelet and antithrombin co-therapies:}

\section{Antiplateletes:}

- Aspirin:

o starting dose $150-500 \mathrm{mg}$ orally

o or IV dose of $250 \mathrm{mg}$ if oral ingestion is not possible

- Clopidogrel:

o Loading dose of $300 \mathrm{mg}$ orally if aged $\leq 75$ years

o Followed by a maintenance dose of $75 \mathrm{mg} /$ daily 


\section{Antithrombin:}

- Unfractionated heparin:

- $60 \mathrm{U} / \mathrm{kg}$ IV bolus with maximum of $4000 \mathrm{U}$ followed by an IV infusion of $12 \mathrm{U} /$ $\mathrm{Kg}$ with a maximum of $1000 \mathrm{U} /$ hours for 24 to 48 hours

o Target aPTT: 50 to 70 s or 1.5 to 2.0 times that of control to be monitored at $3,6,12$ and 24 hours

- Enoxaparin:

o In patients $<75$ years of age:

- $30 \mathrm{mg}$ IV bolus followed 15 minutes later by $1 \mathrm{mg} / \mathrm{kg}$ SC every 12 hours until hospital discharge or maximum of 8 days

- The first two doses should not exceed $100 \mathrm{mg}$

o In patients $>75$ years of age:

- No IV bolus.

- Start with first SC dose of $0.75 \mathrm{mg} \mathrm{kg}$ with a maximum of $75 \mathrm{mg}$ for the first two SC doses

o In patients with creatinine clearance of $<30 \mathrm{~mL} / \mathrm{min}$ regardless of age the SC doses are given once every 24 hours.

- Fondaparinux:

o $2.5 \mathrm{mg}$ IV bolus

o Followed by a SC dose of $2.5 \mathrm{mg}$ once daily up to 8 days or hospital discharge

- Special attention must be given to proper dosing of antithrombotic in elderly and renal failure patients.

\subsection{Assessment of Reperfusion and discharge}

\section{Assessment of reperfusion}

- Relief of symptoms

- Restoration and maintenance of hemodynamic stability

- Restoration and maintenance of electrical stability

Objective assessment of thrombolysis should be carried out by assessing:

1. ST-segment resolution or persistent elevation on the 12-lead ECG

2. Biomarkers

3. Noninvasive imaging

\section{ST-segment Resolution}

Early assessment of ST-segment resolution within $60-90$ minutes after thrombolysis is recommended. 
- Complete resolution $\geq 70 \%$

- Partial resolution $30 \%$ to $70 \%$

- Non resolution $\leq 30 \%$

\section{Biomarkers}

- Cardiac troponin levels 3 days after the onset of symptoms is a good indicator of the infarct size

- CK-MB usually returns to normal within 48 to 72 hours, therefore is a good indicator of recurrent infarctions

\section{Non-invasive imaging}

- Following reperfusion or after the acute phase all patients should have and echocardiography for assessment of infarct size and resting Left Ventricular (LV) function

- Follow up echocardiography is reasonable only after 2 or more months later

- If echocardiography is not feasible MRI may be used as an alternative

- Computed tomography angiography has no role in the routine management of STEMI patients

- For patients with multi-vessel disease, or in whom revascularization of other vessels is considered, stress testing or imaging (eg. Using stress myocardial perfusion scintigraphy, stress echocardiography, positron emission tomography or MRI) for ischemia and viability is indicated.

\section{Transfer and discharge}

- Early transfer back (same day) may be considered in selected, low risk patients after successful primary $\mathrm{PCl}$ without observed dysrhythmia.

- Early discharge after 72 hours approximately is reasonable in selected low risk patients, if early rehabilitation and adequate follow up are arranged.

\subsection{Transfer of Patients with STEMI after thrombolytic therapy}

- Patients with STEMI who has received thrombolytic therapy at a Non-PCl capable hospital should be transferred to a PCl-Capable Hospital for Coronary Angiography (for a reperfusion strategy that combines pharmacological and mechanical methods).

- Immediate transfer to a PCl-capable hospital for coronary angiography is recommended for suitable patients with STEMI who develop cardiogenic shock or acute severe HF, irrespective of the time delay from $\mathrm{MI}$ onset.

- Urgent transfer to a PCl-capable hospital for coronary angiography is reasonable for patients with STEMI who demonstrate evidence of failed reperfusion or reocclusion after fibrinolytic therapy.

- Transfer to a PCl-capable hospital for coronary angiography is reasonable for patients with STEMI who have received fibrinolytic therapy even when hemodynamically stable 
and with clinical evidence of successful reperfusion. Angiography can be performed as soon as logistically feasible at the receiving hospital, and ideally within 24 hours, but should not be performed within the first 2 to 3 hours after administration of fibrinolytic therapy.

- Although individual circumstances will vary, clinical stability is defined by the absence of low output, hypotension, persistent tachycardia, apparent shock, high-grade ventricular or symptomatic supraventricular tachyarrhythmias, and spontaneous recurrent ischemia.

\section{Facilitated PCI}

- Is reperfusion by a combination of immediate pharmacological treatment followed by prompt planned mechanical reperfusion instead of primary $\mathrm{PCl}$.

- This is done to bridge the $\mathrm{PCl}$ related time delay.

- The pharmacological treatment may comprise of full dose lytic therapy, half dose lytic therapy with GP Ilb/llla inhibitor or GP Ilb/llla inhibitor alone.

- However the latest evidence does not show any benefit of facilitated $\mathrm{PCl}$ over primary $\mathrm{PCl}$.

\section{Early PCI (Pharmacoinvasive strategy)}

- By definition this is $\mathrm{PCl}$ after successful lysis within 24 hours of thrombolytic therapy independent of angina and/or ischemia and is also referred to as pharmaco-invasive strategy.

- This strategy requires transferring the patient to a $\mathrm{PCl}$ capable hospital either for immediate "rescue PCl" (see below under 'rescue PCl') when thrombolysis has failed or to perform a non-urgent coronary angiography between 3 to 24 hours after thrombolysis to determine the need for further treatment either in the way of $\mathrm{PCl}$ or CABG.

- Emphasis here should be to achieve very early fibrinolysis ( $<2$ hours)

\section{Rescue PCI}

- $\mathrm{PCl}$ performed in a patient with evidence of failed thrombolysis is called rescue PCI

\section{Indications for rescue $\mathrm{PCl}$ are:}

- Cardiogenic shock in patients less than 75 years of age who are suitable candidates for revascularization.

- Severe congestive heart failure and/or pulmonary edema

- Hemodynamically compromising ventricular arrhythmias

- Cardiogenic shock in patients of 75 years of age or more

- Patients with hemodynamic instability

- Patients with electrical instability

- Patients with persistent ischemic symptoms

- Patients with failed reperfusion and a moderate or large myocardium at risk (anterior)

Delayed PCI - PCl carried out 12 hours to 3 months of STEMI onset 


\subsection{Coronary Artery Bypass Graft (CABG)}

\section{Indications for CABG:}

- Patients with STEMI and coronary anatomy not suitable for PCI who have:

o Ongoing or recurrent ischemia

- Cardiogenic shock

- Severe heart failure

o Other high risk factors

Should undergo urgent CABG

- Patients with STEMI at time of operative repair of mechanical defects

- Aspirin should not be with-held before urgent CABG

- Clopidogrel or ticatrelor should be discontinued at least 24 hours before urgent onpump CABG if possible.

- Eptifibatide and tirofiban should be discontinued at least 2 to 4 hours before urgent CABG

- Abciximab should be discontinued at least 12 hours before urgent CABG

\subsection{Other standard general and/or long term therapeutic measures}

\section{Lipid Management}

- A fasting lipid profile should be performed within 24 hours of symptom onset

- Alternatively could obtain a recent past report from the patient records

- If indicated lipid-lowering medications namely statins should be initiated before discharge

o Treatment goals for LDL-C after STEMI should be $<100 \mathrm{mg} / \mathrm{dL}$

o Further reduction to $<70 \mathrm{mg} / \mathrm{dL}$ appears reasonable

o Dietary advice (see below)

\section{Therapeutic lifestyle changes}

- Should be given to all STEMI patients before discharge

- Dietary advice:
o Diet $2 \mathrm{~g}$ sodium/day
- Low saturated fat ( $<7 \%$ of total calories/day)
o Low cholesterol (<200 mg/day) 
- Other lifestyle modifications:

o Exercise - Encouragement of physical activity

o Weight loss

o Cessation of smoking

- Avoid/Limit/Stop alcohol intake

o Management of comorbidity - tight blood pressure control, Management of diabetes mellitus etc,

\subsection{Wagon Wheel Model for Triage and Transfer of STEMI Patients}

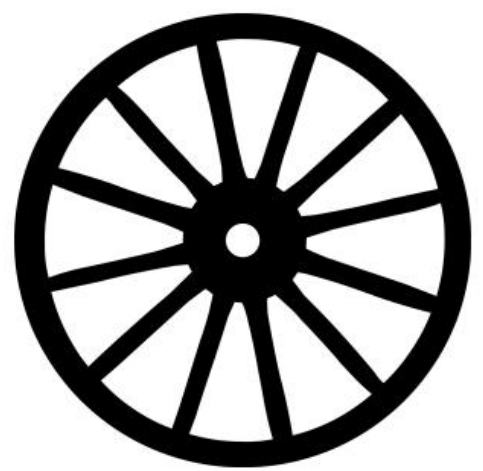

Hub \& Spoke model- Wagon Wheel

\section{Western Province of Sri Lanka}

- 3694 square meters

- 3 districts

- Colombo

- Gampaha

- Kalutara

- Population of 5.8 million.

There are two government hospitals with catheterization laboratories where primary $\mathrm{PCl}$ could be performed.

- Cardiology Institute of NHSL, Colombo

- Sri Jayawardenapura Teaching Hospital, Kotte
Sri Lanka Heart Association (SLHA) and Sri Lanka STEMI forum has introduced the Wagon Wheel Model for Triage and Transfer of STEMI patients using the Hub and Spoke principle.

We plan to implement this model to optimize STEMI care management in each of the Provinces in Sri Lanka.

Below is an example how it could be implemented in the Western Province to optimize the STEMI care system in the Province. 


\section{Wagon Wheel Model}

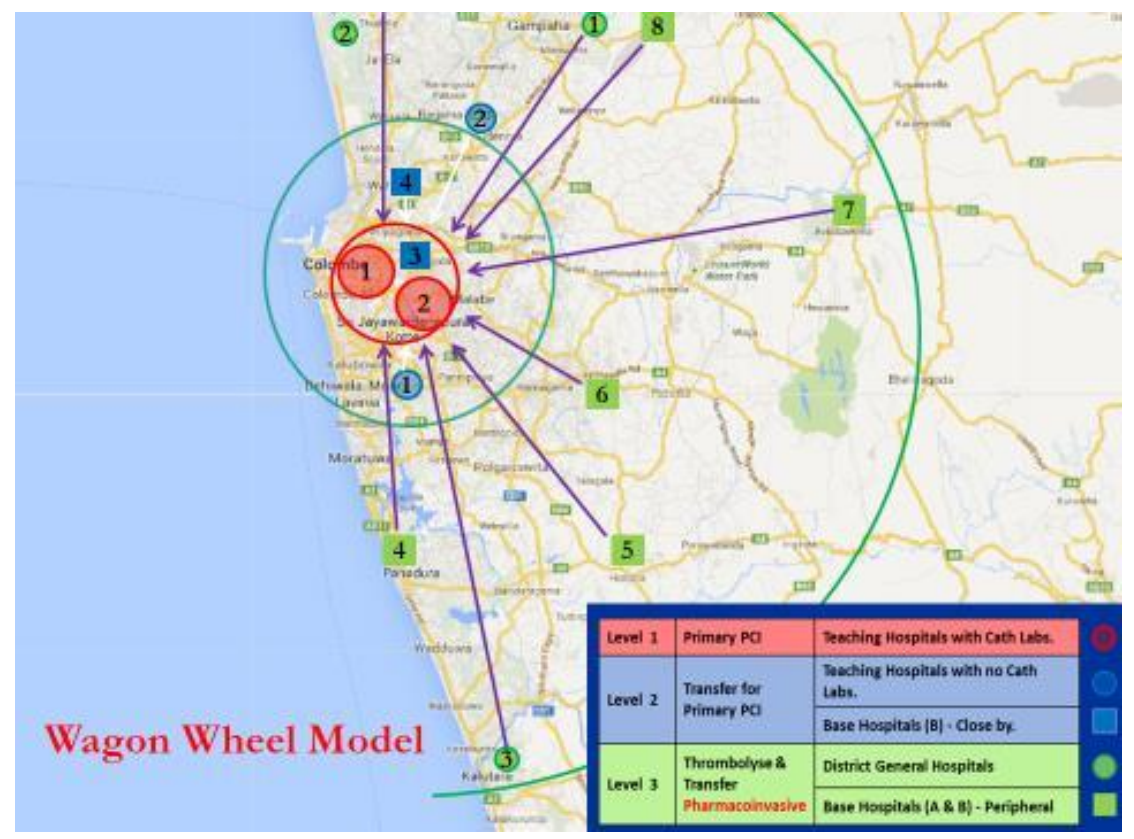

This model has three levels.

- Level 1 - Is the hub with two teaching hospitals with catheterization labs. Where Patients could be directly admitted and primary $\mathrm{PCl}$ could be performed.

- Level 2 - Hospitals in this level would receive patients and will transfer them to the hub for primary $\mathrm{PCl}$

- Level 3 - These hospitals are the furthest away from the hub and therefore will adapt a pharmacoinvasive strategy of reperfusion; i.e., they would treat STEMI patients with thrombolysis and transfer them for angiography with or without $\mathrm{PCl}$.

Level 2 and 3 are determined by the geographical distance from the hub and time taken to transfer to the hub. 


\begin{tabular}{|c|c|c|}
\hline Level & Reperfusion Strategy & Hospitals or type of hospitals \\
\hline $\begin{array}{l}\text { Level } 1 \\
\text { (The Hub) }\end{array}$ & Primary $\mathrm{PCl}$ & $\begin{array}{l}\text { This is the Hub - } 2 \text { Teaching hospitals } \\
\text { with cath. Labs: } \\
\text { - Cardiology Institute of NHSL, } \\
\text { Colombo } \\
\text { - Sri Jayawardenapura Teaching } \\
\text { Hospital, Kotte }\end{array}$ \\
\hline Level 2 & Transfer for Primary PCI & $\begin{array}{l}\text { - Teaching hospitals with no cath labs } \\
\text { - Other Hospitals: } \\
\text { - Base hospitals (Type B) in close } \\
\text { proximity to the hub } \\
\text { - Specialized hospitals or units with } \\
\text { Physicians managing STEMI } \\
\text { close to the hub }\end{array}$ \\
\hline Level 3 & $\begin{array}{l}\text { Thrombolysis } \\
\text { and Transfer - } \\
\text { Pharmacoinvasive } \\
\text { strategy }\end{array}$ & $\begin{array}{l}\text { - District General Hospitals } \\
\text { - Base Hospitals (Both type A \& B) in } \\
\text { the periphery } \\
\text { - Specialized hospitals or units with } \\
\text { Physicians managing STEMI }\end{array}$ \\
\hline
\end{tabular}

\section{Linkages between each of these three levels are built on:}

o Management Protocols for triage and transfer

o Dedicated Transportation System

- Electronic Communication systems

o Telemedicine

o Insurance Re-imbursement when needed

- Real Time Data Collection 


\section{Provincial STEMI Programmes}

This model could be implemented in the other provinces where we already have cath labs in a manner suitable to each province and subsequently to cover the entire island.

\section{Provinces with cath labs:}

1. Western Province

2. Central Province

3. North Central Province

4. Northern Province

5. Southern Province

\section{Provinces without cath labs:}

1. North Central Province

2. Eastern Province

3. Uva Province

4. Sabaragamuwa Province

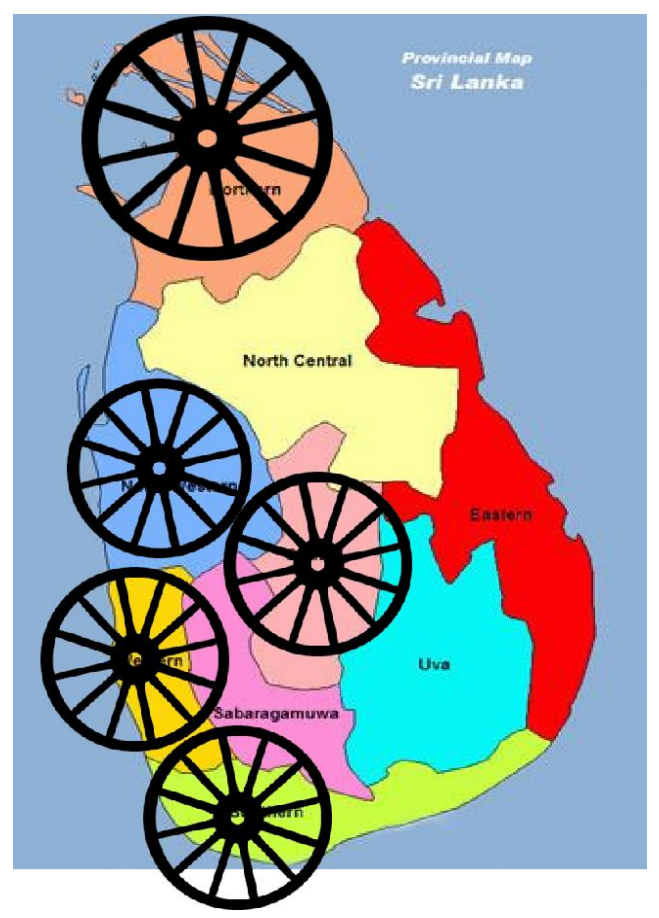

